\title{
Are pre-miR-146a and PTTG1 associated with papillary thyroid cancer?
}

\author{
Marco Marino ${ }^{1,2}$, Valentina Cirello ${ }^{3,4}$, Valentina Gnarini ${ }^{1}$, Carla Colombo ${ }^{3,4}$, \\ Elisa Pignatti ${ }^{1,2}$, Livio Casarini ${ }^{1,2}$, Chiara Diazzi ${ }^{1}$, Vincenzo Rochira ${ }^{1,5}$, Katia Cioni ${ }^{5}$, \\ Bruno Madeo ${ }^{5}$, Cesare Carani ${ }^{1,5}$, Manuela Simoni ${ }^{1,2,5}$ and Laura Fugazzola ${ }^{3,4}$ \\ ${ }^{1}$ Unit and Chair of Endocrinology and Metabolism, NOCSAE, Department of Biomedical, Metabolic and \\ Neural Sciences, University of Modena and Reggio Emilia, Via Pietro Giardini 1355, 41126 Modena, Italy \\ ${ }^{2}$ Center for Genomic Research, University of Modena and Reggio Emilia, Modena, Italy \\ ${ }^{3}$ Department of Clinical Sciences and Community Health, University of Milan, Milan, Italy \\ ${ }^{4}$ Endocrine Unit, Fondazione IRCCS Ca' Granda Milan, Milan, Italy \\ ${ }^{5}$ Azienda USL of Modena, Modena, Italy
}

Correspondence should be addressed to M Simoni

Email

manuela.simoni@unimore.it

\begin{abstract}
Papillary thyroid carcinoma (PTC) is the most common endocrine malignancy, with a steadily increasing incidence in the last few decades worldwide. The predisposition to developing this carcinoma by the heterozygous state of rs 2910164 within the precursor of the miR-146a has been reported, but recently not confirmed. Interestingly, on the same chromosome, almost $50 \mathrm{~kb}$ separate the pre-miR-146a from the pituitary tumor-transforming gene 1 (PTTG1), a proto-oncogene involved in several tumors, including thyroid cancers. In this study, we analyzed, using a case-control design, the genetic association between PTC and the genomic region encompassing pre-miR-146a rs2910164 and PTTG1 rs1862391 and rs2910202. We enrolled 307 affected patients and 206 healthy controls. The possible presence of thyroid nodules in controls was excluded by ultrasonography. All the cases were submitted to singlenucleotide polymorphism (SNP) genotyping of pre-miR-146a and PTTG1, and risk association analyses were carried out. The genotypic and allelic frequencies of pre-miR-146a rs2910164 were not statistically different in the patients and controls, and this SNP was not in linkage disequilibrium with the investigated PTTG1 SNPs. Consistently, meta-analyses, the first including all the affected cases published to date, did not confirm the previously reported association of the heterozygous CG genotype with PTC. The PTTG1 SNPs exhibited the same allelic frequency in the patients and controls and were not associated with the disease. In conclusion, in a well-selected Italian population, neither pre-miR-146a rs2910164 nor PTTG1 rs1862391 and rs2910202 were found to be associated with the risk of developing PTC.
\end{abstract}

Key Words
- PTTG1
- miRNA
- pre-mir-146a
- polymorphism
- SNP
- papillary thyroid carcinoma
- rs2910164
- rs1862391
- rs2910202

Endocrine Connections (2013) 2, 178-185

\section{Introduction}

Thyroid cancers can be well-differentiated, as papillary and follicular subtypes, or poorly differentiated and anaplastic carcinomas. Papillary thyroid carcinoma (PTC) represents up to $80 \%$ of all the thyroid cancer cases.
Various molecular alterations have been reported to be associated with PTC, including BRAF (40-45\%) and RAS (10-20\%) point mutations and RET (10-20\%) or TRK (NTRK1) (<5\%) rearrangements, all involved in the http://www.endocrineconnections.org DOI: 10.1530/EC-13-0066 (c) 2013 The authors Published by Bioscientifica Ltd

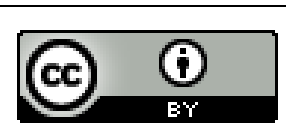

This work is licensed under a Creative Commons Attribution 3.0 Unported License. 
activation of the MAPK pathway (NTRK1) $(1,2)$. Although the literature has a large amount of information on these alterations, there are a few cases of PTC that are not associated with the mutations mentioned above. To fill this gap, the scientific community is still actively searching for new diagnostic and prognostic molecular markers of PTC. In the last 8 years, studies on PTC have been focused on microRNAs (miRNAs), a class of endogenous, small noncoding RNA molecules that function as negative regulators of the expression of protein-encoding genes, involved in many cellular processes such as development, apoptosis, and proliferation $(3,4)$. The first evidence for a potential role for several miRNAs in PTC was the different expression of some of these miRNAs in tumor tissue, compared with unaffected tissue of the thyroid gland (4). In particular, two studies have shown the association between pre-miR-146a rs2910164 and PTC, evaluating the impact of this single-nucleotide polymorphism (SNP) on the regulation of target mRNAs $(5,6)$. As a conclusion, these studies reported an association between the pre-miR$146 a$ CG genomic variant (rs2910164) and the predisposition to developing PTC. However, further case-control studies, including Caucasian and Asian populations, have been unable to confirm this association $(7,8)$. Therefore, the involvement of the genomic variant pre-miR-146a rs2910164 in thyroid cancers remains unclear.

Interestingly, on the same chromosome $5, \sim 50 \mathrm{~kb}$ separate pre-miR-146a (OMIM*610566) from the pituitary tumor-transforming gene 1 (PTTG1) (OMIM*604147), reported to be overexpressed in thyroid carcinomas (9, 10, 11). PTTG1, encoding a securin homolog, is involved in the physiological control of mitosis, DNA repair, gene regulation, and fetal development $(12,13)$. When experimentally mutated, this gene has been shown to promote the proliferation of cancer, to repress the reparation of double-stranded DNA breaks, and to interact with different factors, possibly driving the progression of the disease (13). It has recently been shown that the genomic region on chromosome 5 , encompassing pre-miR$146 a$ and PTTG1, is associated with systemic lupus erythematosus (SLE) $(12,14)$ and cancers $(15,16,17)$, including papillary thyroid tumor $(5,6,7,8,10)$. The aim of the present study was to analyze this region and its association with PTC in a well-selected case-control population of the Northern area of Italy, adopting, as genetic markers, the rs2910164 SNP of pre-miR-146a and, for the first time, the rs1862391 and rs2910201 SNPs of PTTG1. The latter gene was selected both for its involvement in cancers and for its genomic proximity to pre-miR-146a, making it a possible 'true' candidate for the genomic association with PTC previously inconsistently found with pre-miR-146a.

In addition, we carried out meta-analyses on the data reported in the literature $(6,7,8)$, including our data, considering Caucasian and Asian populations.

\section{Subjects and methods}

\section{Subjects}

Using a case-control study design, 307 patients (83 males and 224 females) diagnosed as having PTC and 206 healthy control subjects (75 males and 131 females), all of Caucasian origin and Italian ethnicity, were enrolled. The majority of cases (192/307) were recruited at the Endocrine Unit of the University of Modena and Reggio Emilia, while 115 patients were recruited at the Endocrine Unit of the University of Milan. Tumors were classified according to the thyroid malignancy World Health Organization classification and staged according to the sixth edition of TNM staging (18). Controls, 137/206 from Modena and 69/206 from Milan, were selected among the volunteers. Importantly, to be enrolled, controls had to have a normal thyroid function and a normal ultrasound scan, excluding autoimmune or nodular diseases. The total number of 307 patients and 206 controls satisfied the criteria of the power analysis, requiring a minimum sample size of 164 patients. Written informed consent was obtained from all the participants, who donated a blood sample for association studies on thyroid cancers. The study protocol was approved by the Local Ethics Committee (file no. 122/08).

\section{Criteria for the selection of SNPs}

We selected the rs2910164 SNP of the pre-miR-146a gene because of its direct association with PTC, as reported in the literature $(5,6,7,8)$.

For PTTG1, four different SNPs have been considered in previous studies. Brendle et al. (19) investigated rs1862391, rs1862392, and rs2961952 in breast cancer, whereas Löfgren et al. (12) and Yang et al. (20) described rs2431697 associated with SLE and psoriasis. Based on this information, we decided to analyze the genomic region corresponding to PTTG1, including these four SNPs, but using other, more informative genetic markers following the guidelines of Pettersson et al. (21) for genetic association studies. The online database 'HapMap' (http://hapmap.ncbi.nlm.nih.gov) contains 32 annotated SNPs in the region of over $10 \mathrm{~kb}$ encompassing the whole

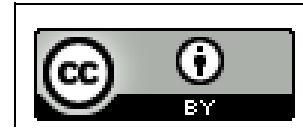

This work is licensed under a Creative Commons Attribution 3.0 Unported License. 
PTTG1 gene. These SNPs were analyzed using Haploview 4.2 (Harvard University and Massachusetts Institute of Technology, USA) to verify those falling within a block of linkage disequilibrium (LD), using the LD parameters $D^{\prime}$ and $r^{2}(21)$. By considering the minor allele frequency (MAF) available in HapMap and the degree of allelic correlation between LD blocks, two SNPs were identified as the best haplotype marker candidates $\left(D^{\prime}=1\right.$ and $r^{2}=1$; Fig. 1A): rs1862391 (A/C) and rs2910202 (C/T). These SNPs exhibit the same MAF, corresponding to the highest MAF among all the SNPs belonging to the PTTG1 LD block (29\% for Utah residents with Northern and Western European ancestry from the CEPH collection, CEU; Fig. 1A). Of these, rs1862391 had been studied previously in breast cancer cases (19). Our analysis revealed several criteria of inadequacy for the other three SNPs studied previously in the literature, such as low probability of LD and different
MAF (between rs1862391 and rs1862392) and the exclusion from LD blocks (rs2961952 and rs2431697). Particularly, the rs2431697 SNP neither belongs to PTTG1 nor falls within the PTTG1 LD block; furthermore, the distance between this SNP and the gene is over $23 \mathrm{~kb}$. The rs1862391 SNP is located at about $500 \mathrm{bp}$ upstream the first exon, inside the promoter region, while the rs2910202 SNP lies about $400 \mathrm{bp}$ downstream exon 3 (between exons 3 and 4). A distance of $2.2 \mathrm{~kb}$ separates the two selected PTTG1 SNPs (Fig. 1A), while a distance of $63.9 \mathrm{~kb}$ separates the rs1862391 SNP of PTTG1 from the rs2910164 SNP of pre-miR-146a (Fig. 1B).

\section{Genomic DNA analysis}

DNA was extracted from the whole peripheral blood of all the patients and controls, using the Nucleon BACC2 Kit
A
B

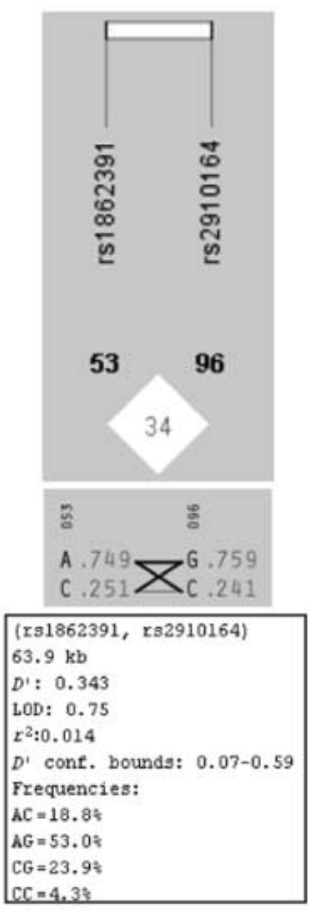

\section{Figure 1}

(A) Haploview window showing the PTTG1 gene and its LD block, containing the two selected SNPs, rs1862391 and rs2910202. The percentage of LD is shown in each diamond. Black diamonds without a number correspond to $100 \%$ of $\operatorname{LD}\left(D^{\prime}=1.0, r^{2}=1\right)$. (B) Results of the analysis of LD between PTTG1 rs1862391 and pre-miR-146a rs2910164 showing a distance of $63.9 \mathrm{~kb}$ between the two SNPs and a very low percentage of $L D, 34 \%\left(D^{\prime}=0.343, r^{2}=0.014\right)$. 
(GE Healthcare, Little Chalfont Buckinghamshire, UK). To genotype the pre-miR-146a rs2910164 SNP, at least $100 \mathrm{ng}$ of DNA were PCR-amplified using specific intronic primers in a One-Advanced Thermocycler (Euroclone S.p.A., Life Sciences Division, Siziano, Italy), according to the following protocol: $98^{\circ} \mathrm{C}$ for 5 min followed by 35 cycles of $98^{\circ} \mathrm{C}$ for $1 \mathrm{~min}, 58^{\circ} \mathrm{C}$ for $1 \mathrm{~min}$, and $72^{\circ} \mathrm{C}$ for $2 \mathrm{~min}$ with a final step of $72^{\circ} \mathrm{C}$ for $10 \mathrm{~min}$. The PCR products were directly sequenced after the removal of the unincorporated dNTPs and primers using ExoSAP-IT (USB Products Affymetrix, Inc., Cleveland, OH, USA). An aliquot of 3-10 ng/100 bp of purified DNA and $3.2 \mathrm{pmol}$ of either the forward or reverse primer was used in standard cycle sequencing reactions with ABI PRISM BigDye Terminators and run on the ABI PRISM 310 Genetic Analyzer (PE Applied Biosystems). The cycle sequencing conditions consisted of 25 cycles of $96^{\circ} \mathrm{C}$ for $30 \mathrm{~s}, 50{ }^{\circ} \mathrm{C}$ for $15 \mathrm{~s}$, and $60{ }^{\circ} \mathrm{C}$ for $4 \mathrm{~min}$. One sequence read from each direction across the entire coding region and including intron-exon boundaries was obtained for each sample. All the primers used for PCR and sequences are listed in Supplementary Table 1, see section on supplementary data given at the end of this article. Electropherograms were analyzed using the Chromas Software (http://technelysium.com.au).

Genotyping for the two PTTG1 SNPs, rs1862391 and rs2910202, was carried out using the High Resolution Melting (HRM) technology on a CFX96 Real-time PCR detection system (Bio-Rad Laboratories). The Beacon Designer 7.91 Software (Bio-Rad) was used to design and test, in silico, all the HRM primers (Supplementary Table 1). To conduct HRM assays, 20 ng of genomic DNA were amplified in a final volume of $15 \mu \mathrm{l}$, containing $1 \times$ SsoFast Eva Green Supermix (Bio-Rad) and $0.5 \mu \mathrm{mol} / 1$ of each primer. The conditions of qPCR were as follows: $98^{\circ} \mathrm{C}$ for 2 min followed by 39 cycles of $3 \mathrm{~s}$ at $98^{\circ} \mathrm{C}$ and $5 \mathrm{~s}$ at $58.2^{\circ} \mathrm{C}$ (for rs1862391) or $58.2^{\circ} \mathrm{C}$ (for rs2910202). After the amplification step, the PCR products were denatured at $98{ }^{\circ} \mathrm{C}$ for $30 \mathrm{~s}$ and slowly renatured at $65^{\circ} \mathrm{C}$ for $90 \mathrm{~s}$. A high-resolution melt was immediately carried out with a progressive denaturation step from 67 to $80^{\circ} \mathrm{C}$ (for rs1862391) or from 66 to $84{ }^{\circ} \mathrm{C}$ (for rs2910202), by increasing the temperature by $0.2^{\circ} \mathrm{C}$ every $10 \mathrm{~s}$. All the HRM raw data (preliminary melting curves) were analyzed using the specific software CFX Manager (Bio-Rad) and Precision Melt Analysis (Bio-Rad). Uncertain results were verified through Sanger sequencing using the 4-capillary ABI Prism 3130 Genetic Analyzer (Applied Biosystems). The FastPCR 6.0 Software (Primer Digital Ltd., Helsinki, Finland) (22) was used to design the PCR and sequence primers for the two PTTG1 SNPs (Supplementary Table 1). Electropherograms were analyzed using the Sequencing Analysis 5.3.1 Software (Applied Biosystems). The accuracy of pre-miR-146a rs2910164 genotyping was assessed by repeating the genotyping analysis for 40 randomly chosen samples. The accuracy of PTTG1 SNP genotyping was assessed by the perfect LD between the two SNPs, as expected in CEU. The success rate of genotyping was 100\%.

\section{Statistical analyses}

To assess the minimum sample size for the present study to be valid, a power analysis was carried out using the GPower 3.1.6 Software (Dusseldorf, Germany) (effect size $w=0.30$; $\alpha=0.05$; power $=0.97$ ), following the programmer's instructions (23). The $\chi^{2}$ test was used to assess the deviation from the Hardy-Weinberg equilibrium (HWE) in the control group and to analyze the differences in genotype distribution and allelic frequencies between the patients and controls. The odds ratio (OR) and its 95\% CI were used to assess the strength of the association between the analyzed polymorphisms and the disease. In metaanalyses, heterogeneity between all the analyses was determined by $I^{2}$, and data with high heterogeneity $\left(I^{2}>\right.$ $50 \%$ ) were processed using a random-effects model or a fixed-effects model (24). All the statistical analyses were carried out using the GraphPad Prism 5 Software (www. graphpad.com), and the Review Manager 5.2 Software (Copenhagen, Denmark) was used for the meta-analyses. $P$ value $<0.05$ was considered to be statistically significant.

\section{Results}

The genotype results did not deviate from the HWE compared with those of the HapMap CEU population ( $P=0.3517$ for pre-miR-146 $a$ and $P=0.4084$ for $P T T G 1)$. In addition, the statistical analyses revealed no differences in genotype distribution between the patients enrolled in Modena and those enrolled in Milan $(P=0.3650$ for pre-miR-146a and $P=0.3684$ for PTTG1). Similarly, no differences were evident between controls recruited at the two sites $(P=0.9193$ for pre-miR-146a and $P=0.8492$ for PTTG1); thus, all the data of patients and controls were assessed together.

No significant differences in genotype distribution and allelic frequencies of pre-miR-146a rs2910164 were found between the patients and controls (Table 1). The OR analysis did not reveal any significant risk association between the CC or GG genotype and PTC (Table 1). The

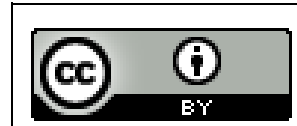

This work is licensed under a Creative Commons Attribution 3.0 Unported License. 
Table 1 Genotype distribution and allelic frequencies of pre-miR-146a rs2910164 in PTC cases and controls.

\begin{tabular}{l} 
rs2910164 \\
\hline Total $n$ \\
Genotype \\
GG \\
CG \\
CC \\
Allele \\
C \\
G
\end{tabular}

\begin{tabular}{l} 
PTC cases $(n(\%))$ \\
\hline 307 \\
$180(58.6)$ \\
$105(34.2)$ \\
$22(7.2)$ \\
$149(24.3)$ \\
$465(75.7)$
\end{tabular}

\begin{tabular}{l}
\hline Controls $(n(\%))$ \\
\hline 206 \\
$105(51)$ \\
$84(40.8)$ \\
$17(8.2)$ \\
$118(28.6)$ \\
$294(71.4)$ \\
\hline
\end{tabular}

$\chi^{2}$
$P=0.2299$
$P=0.1175$

\begin{tabular}{l}
\multicolumn{1}{c}{ Odds ratio $(\%)$} \\
\hline \\
1.363 (GG vs CG + CC) \\
0.755 (CG vs GG + CC) \\
0.8582 (CC vs GG + CG) \\
Reference \\
1.253 (G vs $C)$
\end{tabular}

\begin{tabular}{|c|c|}
\hline $95 \% \mathrm{Cl}$ & $P$ value \\
\hline $0.9556-1.945$ & 0.0869 \\
\hline $0.5243-1.087$ & 0.1302 \\
\hline 0.4439-1.659 & 0.649 \\
\hline $0.9445-1.661$ & 0.1175 \\
\hline
\end{tabular}

previously described association of the CG genotype with PTC $(5,6)$ was not confirmed (Table 1$)$.

All the meta-analyses were carried out by combining the results of the present study with the data of independent association studies from the literature $(6,7,8)$. The meta-analyses did not detect an association either between pre-miR-146a rs2910164 alleles and PTC (Fig. 2A) or between genotypes and PTC (Fig. 2B, C and D).
For PTTG1, the perfect LD between the rs 1862391 and rs2910202 SNPs was confirmed. The genotypic and allelic frequencies of the patients and controls were not different (Table 2). No risk associations were found between the two PTTG1 SNPs and PTC (Table 2). The degree of LD between pre-miR-146a rs2910164 and the chosen PTTG1 SNPs was low (34\%, Fig. 1B).

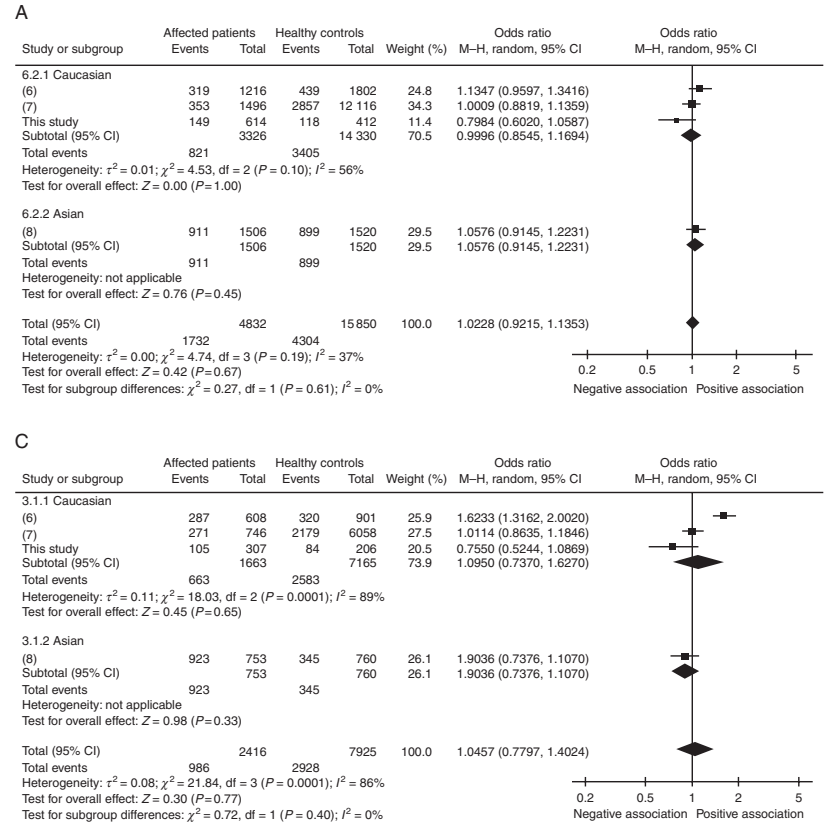

\section{Figure 2}

Results of the meta-analyses for the association risk between pre-miR-146a rs2910164 and PTC. All the meta-analyses included the Caucasian and Asian ethnic subgroups. All the considered studies and all the results of the metaanalyses are represented by the forest plot. The size of the squares indicates the weight of each individual study. The diamond on the top corresponds to the result of the meta-analyses, applied to the Caucasian subgroup Asian subgroup, or both subgroups. $\mathrm{M}-\mathrm{H}$, Mantel-Haenszel method. (A) The plot, showing an OR of 1.0228 , presents the association risk between the alleles (C vs G) of pre-miR-146a rs2910164 and papillary cancer (c) 2013 The authors Published by Bioscientifica Ltd

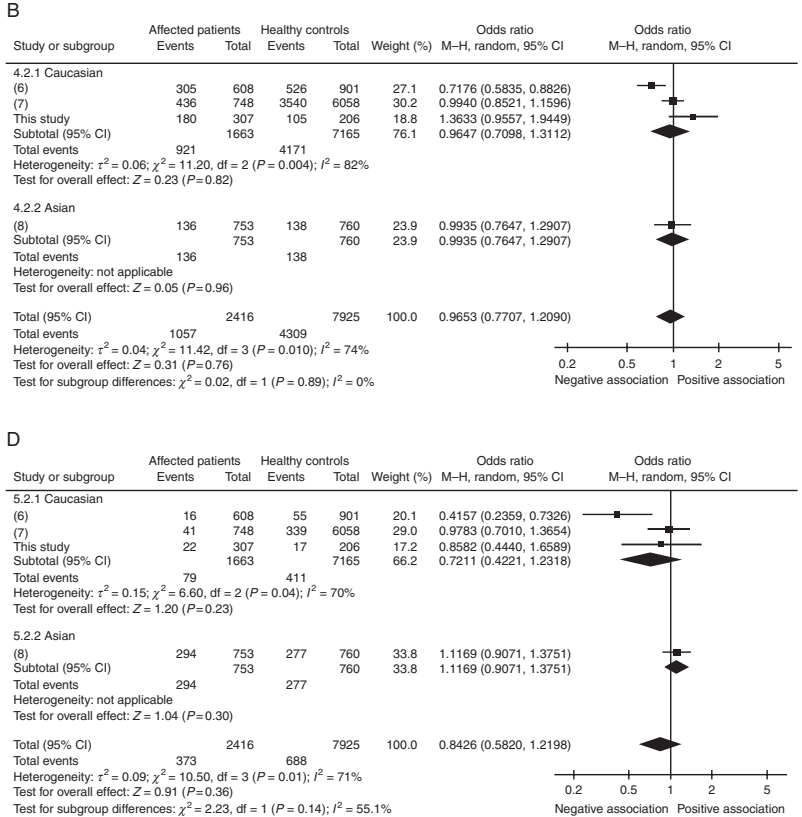

and indicates an association that is not significant. (B) The plot, showing an OR of 0.9653 , presents the association risk between the GG genotype of pre-miR-146a rs2910164 and papillary cancer and indicates an association that is not significant. (C) The plot, showing an OR of 1.0457, presents the association risk between the CG genotype of pre-miR-146a rs2910164 and papillary cancer and indicates an association that is not significant. (D) The plot, showing an OR of 0.8426 , presents the association risk between the CC genotype of pre-miR-146a rs2910164 and papillary cancer and indicates an association that is not significant. http://www.endocrineconnections.org DOI: 10.1530/EC-13-0066
This work is licensed under a Creative Commons Attribution 3.0 Unported License. 
Table 2 Genotype distribution and allelic frequencies of PTTG1 rs1862391 in PTC cases and controls.

\begin{tabular}{|c|c|c|c|c|c|c|}
\hline rs1862391 & PTC cases $(n(\%))$ & Controls $(n(\%))$ & $\chi^{2}$ & Odds ratio & $95 \% \mathrm{Cl}$ & $P$ value \\
\hline Total $n$ & 307 & 206 & & & & \\
\hline Genotype & & & $P=0.9798$ & & & \\
\hline AA & $185(60.7)$ & $123(59.7)$ & & 1.023 (AA vs $A C+C C)$ & $0.7137-1.467$ & 0.9005 \\
\hline$A C$ & $101(32.6)$ & $68(33)$ & & 0.995 (AC vs $A A+C C)$ & $0.6834-1.449$ & 0.9791 \\
\hline $\mathrm{CC}$ & $21(6.7)$ & $15(7.3)$ & & 0.935 (CC vs $A A+A C)$ & $0.4701-1.860$ & 0.8479 \\
\hline Allele & & & $P=0.8541$ & & & \\
\hline $\mathrm{C}$ & $143(23.3)$ & $98(23.8)$ & & Reference & & \\
\hline A & $471(76.7)$ & $314(76.2)$ & & 1.028 (A vs $C$ ) & $0.7660-1.379$ & 0.8541 \\
\hline
\end{tabular}

Since individual genotypes, belonging to two or more SNPs, often were not informative, we extended the analysis by assessing the risk associated with the nine possible diplotypes, arising from the combination of all genotypes of the two SNPs, pre-miR-146a rs2910164 and PTTG1 rs1862391 (Table 3). This analysis did not reveal any specific diplotype to be associated with PTC (Table 3).

\section{Discussion}

In recent years, scientific research has been increasingly orientated to understanding the physiological role and implication of miRNAs in several cancers. Conflicting results exist about the role of pre-miR-146a rs2910164 in thyroid cancers. Indeed, although a significant association between the heterozygous CG genotype of rs2910164 and PTC has been reported, investigating three different Caucasian populations (from Finland, Poland, and USA) (6), these data have not been confirmed in two series of Caucasian (7) and Asian (8) origins. In particular, by comparing patients with benign thyroid tumors with patients with PTC, Wei et al. (8) reported that the GG genotype rather than the CG genotype represented a significant risk factor associated with malignant transformation in a Chinese Han population. In the present study, neither the pre-miR-146a rs2910164 SNP nor the PTTG1 rs1862391 and rs2910201 SNPs were found to be associated with PTC. The differences between the results of the present study and those of the study carried out by Jazdzewski et al. (6) could be due to the different number of patients and controls enrolled, which was lower in the present study. Nevertheless, discordant results have also been obtained in the three larger series published by Jazdzewski et al. (6), Jones et al. (7), and Wei et al. (8), including 608, 748, and 753 PTC cases respectively. Another possibility could lie in the selection of controls, which has previously been carried out based on only anamnestic criteria. By contrast, in the present study, the control subjects were screened and selected on the basis of a normal thyroid ultrasound scan. Owing to the high prevalence of nodular diseases worldwide, and in particular in regions such as Italy, with a mild iodine deficiency, several subjects were excluded due to the incidental demonstration of nodules of any dimension in the absence of clinical signs and symptoms. In particular, we have recently shown that thyroid abnormalities such as nodular goiter and thyroiditis are present in 50.3\% of asymptomatic subjects from in Italy (25). Moreover, differentiated thyroid cancer was found in $1 \%$ of the screened, asymptomatic subjects and in $2 \%$ of those affected by nodular goiter (25). Since previous association studies did not select the control group on the basis of a

Table 3 Odds ratios (ORs) for the risk of developing PTC based on genotype combinations of rs2910164 (pre-miR-146a) and rs1862391 (PTTG1).

\begin{tabular}{|c|c|c|c|c|}
\hline Diplotype & rs2910164 & rs1862391 & PTC cases $(n(\%))$ & Controls $(n(\%))$ \\
\hline Total $n$ & & & 307 & 206 \\
\hline 1 & GG & AA & $101(32.9)$ & $56(27.2)$ \\
\hline 2 & GG & $A C$ & $65(21.2)$ & $40(19.4)$ \\
\hline 3 & GG & $\mathrm{CC}$ & $14(4.6)$ & $9(4.4)$ \\
\hline 4 & CG & $\mathrm{AA}$ & $68(22.1)$ & $55(26.7)$ \\
\hline 5 & CG & $\mathrm{AC}$ & $31(10.1)$ & $24(11.7)$ \\
\hline 6 & CG & $\mathrm{CC}$ & $6(2)$ & $5(2.4)$ \\
\hline 7 & $\mathrm{CC}$ & AA & $16(5.2)$ & $12(5.8)$ \\
\hline 8 & $\mathrm{CC}$ & $A C$ & $5(1.6)$ & 4 (1.9) \\
\hline 9 & $\mathrm{CC}$ & $\mathrm{CC}$ & $1(0.3)$ & $1(0.5)$ \\
\hline
\end{tabular}

\begin{tabular}{lll}
\hline \multicolumn{1}{c}{ OR } & & $\mathbf{9 5 \%} \mathbf{~ C l}$ \\
\cline { 1 - 1 } 1.313 (diplotype 1 vs all) & & $0.8905-1.937$ \\
1.115 (diplotype 2 vs all) & & $0.7174-1.732$ \\
1.046 (diplotype 3 vs all) & & $0.4440-2.464$ \\
0.7811 (diplotype 4 vs all) & & $0.5185-1.177$ \\
0.8518 (diplotype 5 vs all) & & $0.4841-1.499$ \\
0.8013 (diplotype 6 vs all) & & $0.2412-2.662$ \\
0.8889 (diplotype 7 vs all) & & $0.4114-1.921$ \\
0.8361 (diplotype 8 vs all) & & $0.2218-3.152$ \\
0.6699 (diplotype 9 vs all) & & $0.04164-10.78$ \\
\hline
\end{tabular}

$P$ value 
normal thyroid ultrasound scan $(6,7,8)$, it is tempting to speculate that differences in the selection criteria might explain at least in part the discrepancies of the literature.

In such cases, meta-analyses may be helpful. In several published studies, meta-analyses have been carried out to clarify the actual association among polymorphic variants and cancers or other diseases $(24,26,27)$. Recently, this kind of analyses has been carried out for evaluating the association between the pre-miR-146a rs2910164 SNP and over 15 different types of cancers $(26,28,29,30,31$, $32,33)$. In the present study, meta-analyses focused only on the possible association between pre-miR-146a rs2910164 and PTC have been carried out for the first time. Caucasian and Asian ethnic subgroups were considered, separately or together, and the risk association according to the different alleles and genotypes (Fig. 2A, B, $C$ and D) was analyzed. Our meta-analyses included all the studies based on rs2910164 in PTC carried out so far. To the best of our knowledge, no genome-wide association studies on PTC have focused on rs2910164, rs1862391, or rs2910202 so far. Our meta-analyses, including a total of 2416 patients and 7925 controls, did not confirm the association of pre-miR-146a rs2910164 with PTC using any of the models analyzed (dominant or recessive). Therefore, we must conclude that, according to the current knowledge, the genotypes of pre-miR-146a, as assessed in peripheral blood leukocytes, are not associated with PTC (Fig. 2B, C and D).

Given the proximity of PTTG1 to pre-miR-146a and its postulated role in cancers, we extended our analyses to this gene. We reasoned that PTTG1 could be associated with PTC, since a previous study had reported the association of the rs2431697 SNP, located in the intergenic region dividing the PTTG1 and pre-miR-146a genes, with SLE (12). Moreover, several studies have shown the involvement of PTTG1 in the onset and/or progression of different types of tumors $(10,11,13,15,16,17)$, including thyroid cancers (10). The postulated role of securin, the protein encoded by PTTG1, in thyroid tumorigenesis and in the development of hyperplastic lesions could be due to its overexpression during metaphase-anaphase transition (10), which could generate aneuploidy $(10,13)$. Taking all these data into consideration, we decided to explore the association of PTTG1 with PTC, using, for the first time, the rs1862391 A/C and rs2910201 C/T SNPs as genetic markers. The PTTG1 SNPs (rs1862391 and rs2910202), in perfect LD, exhibited the same allelic frequency in patients and controls and were not associated with PTC, probably excluding a possible role of this gene in thyroid tumorigenesis.
In conclusion, the present case-control study, in a well-selected population of the Northern area of Italy, and the meta-analyses, combining the results of all the association studies carried out so far, demonstrate that pre-miR-146a rs2910164 is not associated with PTC. In particular, no genotype was found to be associated with an increased risk of developing PTC, including the previously suggested heterozygous CG genotype. Extending the genetic analysis to the contiguous genomic region, we showed that PTTG1 was not associated with PTC. In the light of these data, the genomic region encompassing pre-miR-146a and PTTG1 is probably not associated with the predisposition to developing PTC. The occurrence of PTTG1 gene mutations in PTC patients and the role of somatic mutations of both the investigated genes remain to be explored.

\section{Supplementary data}

This is linked to the online version of the paper at http://dx.doi.org/10.1530/ EC-13-0066.

\section{Declaration of interest}

The authors declare that there is no conflict of interest that could be perceived as prejudicing the impartiality of the research reported.

\section{Funding}

This work was supported by Fondazione Cassa di Risparmio di Modena (grant number 2006/0320).

\section{Author contribution statement}

M Marino and V Cirello contributed equally to this work.

\section{References}

1 Romei C, Fugazzola L, Puxeddu E, Frasca F, Viola D, Muzza M, Moretti S, Nicolosi ML, Giani C, Cirello V et al. Modifications in the papillary thyroid cancer gene profile over the last 15 years. Journal of Clinical Endocrinology and Metabolism 201297 1758-1765. (doi:10.1210/jc. 2012-1269)

2 Soares P, Lima J, Preto A, Castro P, Vinagre J, Celestino R, Couto JP, Prazeres H, Eloy C, Báximo V et al. Genetic alterations in poorly differentiated and undifferentiated thyroid carcinomas. Current Genomics 201112 609-617. (doi:10.2174/138920211798120853)

3 de la Chapelle A \& Jazdzewski K. MicroRNAs in thyroid cancer. Journal of Clinical Endocrinology and Metabolism 201196 3326-3336. (doi:10.1210/jc.2011-1004)

4 He H, Jazdzewski K, Li W, Liyanarachchi S, Nagy R, Volinia S, Calin GA, Liu CG, Franssila K, Suster S et al. The role of microRNA genes in papillary thyroid carcinoma. PNAS 2005102 19075-19080. (doi:10.1073/pnas.0509603102)

5 Jazdzewski K, Liyanarachchi S, Swierniak M, Pachucki J, Ringel MD, Jarzab B \& de la Chapelle A. Polymorphic mature microRNAs from

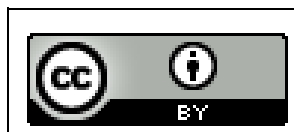


passenger strand of pre-miR-146a contribute to thyroid cancer. PNAS 2009106 1502-1505. (doi:10.1073/pnas.0812591106)

6 Jazdzewski K, Murray EL, Franssila K, Jarzab B, Schoenberg DR \& de la Chapelle A. Common SNP in pre-miR-146a decreases mature miR expression and predisposes to papillary thyroid carcinoma. PNAS 2008105 7269-7274. (doi:10.1073/pnas.0802682105)

7 Jones AM, Howarth KM, Martin L, Gorman M, Mihai R, Moss L, Auton A, Lemon C, Mehanna H, Mohan $\mathrm{H}$ et al. Thyroid cancer susceptibility polymorphisms: confirmation of loci on chromosomes $9 \mathrm{q} 22$ and $14 \mathrm{q} 13$, validation of a recessive $8 \mathrm{q} 24$ locus and failure to replicate a locus on 5q24. Journal of Medical Genetics 201249 158-163. (doi:10.1136/jmedgenet-2011-100586)

8 Wei WJ, Wang YL, Li DS, Wang Y, Wang XF, Zhu YX, Yang YJ, Wang ZY, Ma YY, Wu Y et al. Association between the rs2910164 polymorphism in pre-Mir-146a sequence and thyroid carcinogenesis. PLOS ONE 20138 e56638. (doi:10.1371/journal.pone.0056638)

9 Hsueh C, Lin JD, Chang YS, Hsueh S, Chao TC, Yu JS, Jung SM, Tseng NM, Sun JH, Kuo SY et al. Prognostic significance of pituitary tumour-transforming gene-binding factor (PBF) expression in papillary thyroid carcinoma. Clinical Endocrinology 201378 303-309. (doi:10.1111/cen.12007)

10 Lewy GD, Sharma N, Seed RI, Smith VE, Boelaert K \& McCabe CJ. The pituitary tumor transforming gene in thyroid cancer. Journal of Endocrinological Investigation 201235 425-433. (doi:10.3275/8332)

11 Smith VE, Franklyn JA \& McCabe CJ. Pituitary tumor-transforming gene and its binding factor in endocrine cancer. Expert Reviews in Molecular Medicine 201012 e38. (doi:10.1017/S1462399410001699)

12 Löfgren SE, Frostegård J, Truedsson L, Pons-Estel BA, D'Alfonso S, Witte T, Lauwerys BR, Endreffy E, Kovács L, Vasconcelos C et al. Genetic association of miRNA-146a with systemic lupus erythematosus in Europeans through decreased expression of the gene. Genes and Immunity 201213 268-274. (doi:10.1038/gene.2011.84)

13 Mora-Santos M, Castilla C, Herrero-Ruiz J, Giráldez S, Limón-Mortés MC, Sáez C, Japón MÁ, Tortolero M \& Romero F. A single mutation in Securin induces chromosomal instability and enhances cell invasion. European Journal of Cancer 201349 500-510. (doi:10.1016/j.ejca.2012.06.024)

14 Harley JB, Alarcon-Riquelme ME, Criswell LA, Jacob CO, Kimberly RP, Moser KL, Tsao BP, Vyse TJ, Langefeld CD, Nath SK et al. Genome-wide association scan in women with systemic lupus erythematosus identifies susceptibility variants in ITGAM, PXK, KIAA1542 and other loci. Nature Genetics 200840 204-210. (doi:10.1038/ng.81)

15 Chen S, Xiao L, Liu Z, Liu J \& Liu Y. Pituitary tumor transforming gene-1 haplotypes and risk of pituitary adenoma: a case-control study. BMC Medical Genetics 201112 44. (doi:10.1186/1471-2350-12-44)

16 Wondergem B, Zhang Z, Huang D, Ong CK, Koeman J, Hof DV, Petillo D, Ooi A, Anema J, Lane B et al. Expression of the PTTG1 oncogene is associated with aggressive clear cell renal cell carcinoma. Cancer Research 201272 4361-4371. (doi:10.1158/0008-5472.CAN11-2330)

17 Yoon CH, Kim MJ, Lee H, Kim RK, Lim EJ, Yoo KC, Lee GH, Cui YH, Oh YS, Gye MC et al. PTTG1 oncogene promotes tumor malignancy via epithelial to mesenchymal transition and expansion of cancer stem cell population. Journal of Biological Chemistry 2012287 19516-19527. (doi:10.1074/jbc.M111.337428)

18 Greene FL, Page DL, Fleming ID, April F, Balch CM, Haller DG \& Monica M. In American Joint Committee of Cancer, Cancer Staging Manual, 6th edn, pp 5-8. Philadelphia, PA, USA: Springer, 2002.

19 Brendle A, Brandt A, Johansson R, Enquist K, Hallmans G, Hemminki K, Lenner P \& Försti A. Single nucleotide polymorphisms in chromosomal instability genes and risk and clinical outcome of breast cancer: a Swedish prospective case-control study. European Journal of Cancer 200945 435-442. (doi:10.1016/j.ejca.2008.10.001)

20 Yang Q, Liu H, Qu L, Fu X, Yu Y, Yu G, Tian H, Yu Y, Sun D, Peng et al. Investigation of 20 non-HLA (human leucocyte antigen) psoriasis susceptibility loci in Chinese patients with psoriatic arthritis and psoriasis vulgaris. British Journal of Dermatology 2013168 1060-1065. (doi:10.1111/bjd.12142)

21 Pettersson FH, Anderson CA, Clarke GM, Barrett JC, Cardon LR, Morris AP \& Zondervan KT. Marker selection for genetic case-control association studies. Nature Protocols 20094 743-752. (doi:10.1038/ nprot.2009.38)

22 Kalendar R, Lee D \& Schulman AH. FastPCR software for PCR primer and probe design and repeat search. Genes, Chromosomes \& Cancer 2009 3 1-14.

23 Faul F, Erdfelder E, Lang AG \& Buchner A. G*Power 3: a flexible statistical power analysis program for the social, behavioral, and biomedical sciences. Behavior Research Methods 200739 175-191. (doi:10.3758/BF03193146)

24 Chen HF, Hu TT, Zheng XY, Li MQ, Luo MH, Yao YX, Chen Q \& Yu SY. Association between miR-146a rs2910164 polymorphism and autoimmune diseases susceptibility: a meta-analysis. Gene $2013 \mathbf{5 2 1}$ 259-264. (doi:10.1016/j.gene.2013.03.073)

25 Gnarini VL, Brigante G, Della Valle E, Diazzi C, Madeo B, Carani C, Rochira V \& Simoni M. Very high prevalence of ultrasound thyroid scan abnormalities in healthy volunteers in Modena, Italy. Journal of Endocrinological Investigation 2013 [in press].

26 Srivastava K \& Srivastava A. Comprehensive review of genetic association studies and meta-analyses on miRNA polymorphisms and cancer risk. PLOS ONE 20127 e50966. (doi:10.1371/journal.pone. 0050966)

27 Tian T, Xu Y, Dai J, Wu J, Shen H \& Hu Z. Functional polymorphisms in two pre-microRNAs and cancer risk: a meta-analysis. International Journal of Molecular Epidemiology and Genetics 20101 358-366.

28 He B, Pan Y, Cho WC, Xu Y, Gu L, Nie Z, Chen L, Song G, Gao T, Li R et al. The association between four genetic variants in microRNAs (rs11614913, rs2910164, rs3746444, rs2292832) and cancer risk: evidence from published studies. PLoS ONE 20127 e49032. (doi:10.1371/journal.pone.0049032)

29 Qiu LX, He J, Wang MY, Zhang RX, Shi TY, Zhu ML, Mao C, Sun S, $\mathrm{Lv} F \mathrm{FF}$, Zheng CL et al. The association between common genetic variant of microRNA-146a and cancer susceptibility. Cytokine 201156 695-698. (doi:10.1016/j.cyto.2011.09.001)

30 Wang J, Wang Q, Liu H, Shao N, Tan B, Zhang G, Wang K, Jia Y, Ma W, Wang $\mathrm{N}$ et al. The association of miR-146a rs2910164 and miR-196a2 rs11614913 polymorphisms with cancer risk: a meta-analysis of 32 studies. Mutagenesis 201227 779-788. (doi:10.1093/mutage/ges052)

31 Xu W, Xu J, Liu S, Chen B, Wang X, Li Y, Qian Y, Zhao W \& Wu J. Effects of common polymorphisms rs11614913 in miR-196a2 and rs2910164 in miR-146a on cancer susceptibility: a meta-analysis. PLoS ONE 20116 e20471. (doi:10.1371/journal.pone.0020471)

32 Xu Y, Gu L, Pan Y, Li R, Gao T, Song G, Nie Z, Chen L, Wang S \& He B. Different effects of three polymorphisms in microRNAs on cancer risk in Asian population: evidence from published literatures. PLOS ONE 20138 e65123. (doi:10.1371/journal.pone.0065123)

33 Yin Z, Yan L, Cui Z, Li X, Ren Y \& Zhou B. Effects of common polymorphisms rs2910164 in miR-146a and rs3746444 in miR-499 on cancer susceptibility: a meta-analysis. Molecular Biology Reports 2013 40 3003-3013. (doi:10.1007/s11033-012-2372-7)

Received in final form 19 September 2013 Accepted 2 October 2013 http://www.endocrineconnections.org

DOI: 10.1530/EC-13-0066 (c) 2013 The authors Published by Bioscientifica Ltd

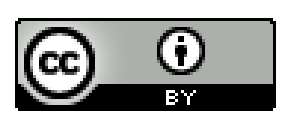

This work is licensed under a Creative Commons Attribution 3.0 Unported License. 